\title{
Predictive Evaluation on Cytological Sample of Metastatic Melanoma: The Role of BRAF Immunocytochemistry in the Molecular Era
}

\author{
Andrea Ronchi ${ }^{1, *}$, Marco Montella ${ }^{1}$, Federica Zito Marino ${ }^{1}$, Michele Caraglia ${ }^{\text {ID }}$, Anna Grimaldi ${ }^{2}$, \\ Giuseppe Argenziano $^{3}$ (D), Elvira Moscarella ${ }^{3}$, Gabriella Brancaccio ${ }^{3}$, Teresa Troiani ${ }^{4}$, Stefania Napolitano ${ }^{4}$, \\ Renato Franco ${ }^{1, *(\mathbb{D})}$ and Immacolata Cozzolino ${ }^{1}$
}

Citation: Ronchi, A.; Montella, M.; Zito Marino, F.; Caraglia, M.; Grimaldi, A.; Argenziano, G.; Moscarella, E.; Brancaccio, G.; Troiani, T.; Napolitano, S.; et al. Predictive Evaluation on Cytological Sample of Metastatic Melanoma: The Role of BRAF Immunocytochemistry in the Molecular Era. Diagnostics 2021, 11, 1110. https://doi.org/10.3390/ diagnostics11061110

Academic Editor: Thomas Botton

Received: 18 April 2021

Accepted: 16 June 2021

Published: 18 June 2021

Publisher's Note: MDPI stays neutral with regard to jurisdictional claims in published maps and institutional affiliations.

Copyright: (c) 2021 by the authors. Licensee MDPI, Basel, Switzerland. This article is an open access article distributed under the terms and conditions of the Creative Commons Attribution (CC BY) license (https:/ / creativecommons.org/licenses/by/ $4.0 /)$.
1 Pathology Unit, Department of Mental and Physical Health and Preventive Medicine, Università degli Studi della Campania “Luigi Vanvitelli”, 80138 Naples, Italy; marco.montella@unicampania.it (M.M.); federica.zitomarino@unicampania.it (F.Z.M.); coimma73@gmail.com (I.C.)

2 Department of Precision Medicine, University of Campania “Luigi Vanvitelli", 80138 Naples, Italy; michele.caraglia@unicampania.it (M.C.); anna.grimaldi@unicampania.it (A.G.)

3 Dermatology Unit, Department of Mental and Physical Health and Preventive Medicine, Università degli Studi della Campania “Luigi Vanvitelli”, 80138 Naples, Italy; giuseppe.argenziano@unicampania.it (G.A.); elvira.moscarella@unicampania.it (E.M.); gabri.brancaccio@gmail.com (G.B.)

4 Oncology Unit, Department of Precision Medicine, Università della Campania "Luigi Vanvitelli", 80131 Naples, Italy; teresa.troiani@unicampania.it (T.T.); stefania.napolitano@unicampania.it (S.N.)

* Correspondence: andrea.ronchi@unicampania.it (A.R.); renato.franco@unicampania.it (R.F.)

Abstract: Background: Cutaneous malignant melanoma is an aggressive neoplasm. In advanced cases, the therapeutic choice depends on the mutational status of BRAF. Fine needle aspiration cytology (FNA) is often applied to the management of patients affected by melanoma, mainly for the diagnosis of metastases. The evaluation of BRAF mutational status by sequencing technique on cytological samples may be inconvenient, as it is a time and biomaterial-consuming technique. Recently, BRAF immunocytochemistry (ICC) was applied for the evaluation of BRAF V600E mutational status. Although it may be useful mainly in cytological samples, data about BRAF ICC on cytological samples are missing. Methods: We performed BRAF ICC on a series of 50 FNA samples of metastatic melanoma. BRAF molecular analysis was performed on the same cytological samples or on the corresponding histological samples. Molecular analysis was considered the gold standard. Results: BRAF ICC results were adequate in 49 out of $50(98 \%)$ cases, positive in 15 out of $50(30 \%)$ cases and negative in 34 out of $50(68 \%)$ of cases. Overall, BRAF ICC sensitivity, specificity, positive predictive value and negative predictive value results were $88.2 \%, 100 \%, 100 \%$ and $94.1 \%$, respectively. The diagnostic performance of BRAF ICC results was perfect when molecular evaluation was performed on the same cytological samples. Hyperpigmentation represents the main limitation of the technique. Conclusions: BRAF ICC is a rapid, cost-effective method for detecting BRAF V600E mutation in melanoma metastases, applicable with high diagnostic performance to cytological samples. It could represent the first step to evaluate BRAF mutational status in cytological samples, mainly in poorly cellular cases.

Keywords: melanoma; fine needle aspiration; BRAF; immunocytochemistry

\section{Introduction}

Cutaneous melanoma (CM) is the most aggressive skin neoplasm, responsible for approximately 61,000 deaths per year worldwide [1]. Indeed, CM shows an early tendency to metastasize; thus, lymphatic and hematogenous metastases occur early in tumor progression and the 5 -year overall survival (OS) is $23 \%$ for advanced melanoma [2]. Beyond cases of clear advanced disease obtained by imaging, the diagnosis of localized or regional recurrence could be very difficult [3]. FNA is a reliable tool to diagnose CM metastases, as it is a 
rapid and cheap technique [4]. Metastatic CM incidence is less than 5 cases per 100,000 per year [5]. The diagnosis of CM metastases is often based on clinical and instrumental findings and a direct sampling is usually unnecessary. Moreover, deep visceral CM metastases may be difficult to sample by percutaneous techniques such as FNA. So, few studies have evaluated the application of FNA to metastatic CM. However, some reports have shown that the use of FNA with image guidance demonstrates excellent diagnostic performance for the diagnosis of CM metastases, being that the sensitivity (SE) and specificity (SP) range is between $86.5 \%$ and $100.0 \%$ [3]. A correct sampling of the lesion and adequate cellularity is mandatory to optimize the diagnostic performance of FNC. Particularly, false-negative cases could be related to inadequate sampling. In addition, above all in the cases of a suspicious metastatic site of difficult access, the low cellularity of the samples containing only scattered malignant cells does not permit additional ancillary methods for better characterization [3]. However, when a diagnosis of metastatic CM is posed, the current therapeutic approach is based on advanced medical treatment. Indeed, historically, surgery was the only therapy for $\mathrm{CM}$, and therapeutic options were not available for patients with advanced disease. In the last few years, a better understanding of the molecular landscape of $\mathrm{CM}$ led to the development of new therapies for advanced disease. Mitogen-activated protein kinase (MAPK) pathway deregulation has emerged as a main molecular event in the $\mathrm{CM}$ oncogenesis, present in up to $70-80 \%$ of cases [6]. It is actually known that about $40-50 \%$ of $\mathrm{CM}$ cases harbor the mutation of the gene for v-Raf murine sarcoma viral oncogene homolog $\mathrm{B}$ (BRAF), leading to the BRAF protein constitutive activation, a serine/threonine-protein kinase in the MAPK signaling pathway [7]. The most frequent BRAF mutation is V600E, accounting for approximately $70-80 \%$ of cases, followed by V600K, V600D and V600R [7,8]. The identification of BRAF mutations has a predictive value in advanced $\mathrm{CM}$, leading to the selection of patients who may benefit, in the presence of a V600 mutation, from treatment with MAPK inhibitors [9]. Therefore, the molecular workflow for CM should start with the identification of BRAF mutation in stage IIIC or IV $\mathrm{CM}$ patients [9]. This evaluation can be carried out using different molecular procedures, including direct sequencing of the PCR product, pyrosequencing, RealTime PCR, molecular hybridization on filter and mass spectrometry [10]. Recently, a BRAF V600E mutationspecific immunohistochemical antibody was introduced, with sensitivity and specificity comparable to the molecular tests in histological samples [11-13]. It was consequently proposed, and applied, as a screening tool for a rapid and cheaper assessment of BRAF V600E mutational status in histological samples [11-14]. In the clinical setting of the advanced disease, the biomaterial obtained from metastasis through FNA procedure for diagnosis could be also used for the assessment of the current BRAF status of the patients, who cannot benefit from surgery with radical intent, avoiding unnecessary surgical stress and public health charges [15-17]. Indeed, although the BRAF status usually does not change in the metastasis with respect to the primitive $\mathrm{CM}$, a recent metanalysis underlined the discrepancy between the primitive $\mathrm{CM}$ and relative metastasis, calculating a change from BRAF mutation in primitive CM to wild type in relative metastasis in $15.1 \%$ of analyzed series and an opposite change in $13.2 \%$ of such series [18]. In addition, metastasis could be the only clinical feature of melanoma with an unknown BRAF status. Thus, cytological samples could represent the only biomaterials useful for detecting BRAF status $[16,17]$. Finally, a different BRAF status of the metastasis with respect to known primitive tumors could also be the expression of another tumor with a different mutational status [19]. For all these reasons, the recommendation of the current guidelines is to determine the mutational status of the metastasis, when possible [20].

However, some limitations about the use of cytological samples for predictive purposes should be addressed. First, the largest limitation to obtain reliable results is the number of neoplastic cells in the cytological sample, sometimes unsatisfactory also for diagnostic purposes [18]. Thus, in such cases, it is required to resort to a surgical sampling of the metastasis for the BRAF status assessment, with further stress for the patient, or to the primitive tumor $[21,22]$. In addition, in the last case, not only is the BRAF status 
not always representative of the current status, but when the primitive tumor is a thin melanoma, the number of neoplastic cells may not be enough for the assessment of BRAF mutation with classical extractive methods. Indeed, the immunohistochemical evaluation requires a relatively smaller amount of neoplastic cells, if compared to molecular extractive methods for mutation detections [11-14]. Thus, in the primitive CM, when very thin or previously highly consumed for diagnosis, BRAF immunohistochemical detection could offer higher diagnostic accuracy than molecular testing [11-14]. A further limitation is related to the complete consumption of the direct smears of the metastasis cytological sample for DNA extraction, with loss of archival biological material. Finally, molecular analysis is expensive, requires experienced technicians and is not widespread in all laboratories worldwide. Thus, although the application of molecular analysis to cytological samples is generally successful, the use of immunocytochemistry (ICC) for predictive tests in advanced CM on cytological samples could present some advantages.

The aim of our study is the evaluation of the diagnostic performance of BRAF ICC on a retrospective series of metastatic CM diagnosed by FNA, comparing ICC results with the gold standard molecular analysis results.

\section{Materials and Methods}

\subsection{Patients Selection}

All cases of US-guided or CT-guided FNA in patients with previous CM and clinical suspicious metastasis in the follow-up between January 2017 and December 2020 from the archives of the Pathology Unit of University "Vanvitelli" Hospital in Naples (Italy) were revised. Negative cases were excluded. Positive cases were selected in our series according to the following criteria: (1) diagnosis of CM metastases rendered on FNA samples; (2) the realization of a cell-block (CB); (3) the presence of residual biomaterial in the CB; (4) molecular evaluation of BRAF mutational status performed on the same cytological sample or the corresponding histological sample, when surgery was performed.

Fifty-seven positive consecutive cases were initially retrieved. All the cases were reviewed by two expert cytopathologists (MM, IC) to confirm the diagnosis and to assess the presence of sufficient residual tumor cells in the CB. The diagnosis was confirmed in all cases. Two cases were excluded because they did not include a $C B$, and 4 cases were excluded because the CB did not include residual biomaterial. One case was excluded because a molecular evaluation was not possible, as this case only included few neoplastic cells. Therefore, the final number of cases included in the series was 50. Each case included direct smears-stained by Diff Quick and Papanicolaou and a CB. Clinical and molecular data were collected from the archives of the Pathology Unit.

\subsection{BRAF V600E Immunocytochemistry Technique}

ICC was performed on $4 \mu \mathrm{m}$-thick FFPE cell-block slices using a fully automatized assay based on the Ventana ${ }^{\circledR}$ BRAF V600E (VE1, Ventana-Roche Diagnostics, Meylan, France) mouse monoclonal primary antibody in combination with the Ventana OptiView DAB IHC Detection Kit ${ }^{\circledR}$ on the Ventana ${ }^{\circledR}$ Benchmark XT platform (Ventana-Roche Diagnostics, Meylan, France). The procedure was performed according to the manufacturer's instructions.

\subsection{BRAF Immunocytochemistry Evaluation}

All immunostained slides were evaluated by two cytopathologists in absence of any information about molecular data. Immunostaining was primarily interpreted as positive or negative. We defined a case as positive if it showed diffuse cytoplasmic staining, according to data reported in histological series $[13,23]$. We considered a case as negative if no staining or only nuclear dot staining was present. Furthermore, the percentage of positive neoplastic cells and intensity of the staining were recorded. The percentage of positive neoplastic cells were calculated by comparing the stained neoplastic cells to the total number of neoplastic cells in the slide. 


\subsection{BRAF Molecular Analysis}

According to strategies followed during clinical management, molecular analyses were previously performed preferentially on cytological samples; when the number of neoplastic cells was not enough for extractive-based molecular testing, the analysis was conducted on the histological sample of the $\mathrm{CM}$ metastasis when the patients were submitted to surgery or on the primitive tumors if tissue from metastases were not available.

The evaluation of the mutational status of the BRAF gene was performed by the NGS method. DNA was extracted from 4 unstained $10 \mu \mathrm{M}$ FFPE tissue sections or from the cytological samples. DNA was obtained using the QIAamp ${ }^{\circledR}$ DNA FFPE kit Tissue (Qiagen, Hilden, Germany) for histological samples or using the Qiagen QIAamp ${ }^{\circledR}$ DNA Micro kit. (Qiagen, Hilden, Germany) for cytological samples, according to the manufacturer's instructions. The massive parallel sequencing of DNA libraries by ION Torrent Personal was used as previously reported [7]. Sequencing was carried out using different chips on the Ion Personal Genome Machine System (PGM ${ }^{\mathrm{TM}}$, Thermo Fisher Scientific, Waltham, MA, USA). Torrent Suite Software v.4.0.2 (Life Technologies, Carlsbad, CA, USA) to assess run performance and data analysis was used. Integrative Genomics Viewer (IGV v 2.2, Broad Institute, Cambridge, MA, USA) was used for visual inspection of the aligned reads. Data were analyzed using Ion Reporter software [22] and further filtered through quality checking. We selected all SNVs in the studied genes resulting in a non-synonymous amino acid change, or a premature stop codon, and all short indels resulting in either a frameshift or insertion/deletion of amino acids. All SNVs were analyzed for previously reported hotspot mutations (somatic mutations reported in COSMIC database) and novel variations, i.e., new mutations detected by NGS but not reported in either COSMIC or db SNP databases.

\subsection{Evaluation of Diagnostic Performance of BRAF Immunocytochemistry}

BRAF ICC SE and SP were calculated considering the molecular evaluation of BRAF mutational status as the gold standard.

\subsection{Ethical Consideration}

The present study was retrospectively conducted on archival biological samples. Both the cytological and histological diagnoses, as well as molecular analysis of BRAF mutational status, had already been rendered in all cases. At the time of the FNA procedure, a written consent, including the consent to use the diagnostic data for scientific purposes, had been obtained from each patient. The approval by the institutional ethical board was collected.

\section{Results}

\subsection{Clinic-Pathological Data}

This series included 50 cases diagnosed as $\mathrm{CM}$ metastases on cytological samples obtained by FNA. The series included $38(76 \%)$ males and $12(24 \%)$ females (M:F = 3.2:1), with a mean age of 62 years (range from 38 to 86 years). The $\mathrm{CM}$ metastases were located at lymph nodes (LNs) in 43 out of $50(86 \%)$ of cases, at subcutaneous nodules in 4 out of 50 $(8 \%)$ of cases and at lungs in the remaining $3(6 \%)$ of cases. In detail, the location of $\mathrm{CM}$ metastases was: right axillary LNs in 14 cases, left axillary LNs in 8 cases, right inguinal LNs in 8 cases, left inguinal LNs in 4 cases, right cervical LNs in 5 cases, left intraparotid LNs in 2 cases, right intraparotid LN in 1 case, deep para-aortic LN in 1 case, right lung in 2 cases, left lung in 1 case, subcutis in 4 cases. Clinical findings are summarized in Table 1. 
Table 1. Clinical findings.

\begin{tabular}{cc}
\hline Total Cases & 50 \\
Sex & $38(76 \%)$ \\
Males & $12(24 \%)$ \\
Females & $3.2: 1$ \\
Males:Females & \\
Age & $38-86$ \\
Range & 62 \\
Mean age & \\
Location & $14(28 \%)$ \\
Right axillary lymph node & $8(16 \%)$ \\
Left axillary lymph node & $8(16 \%)$ \\
Right inguinal lymph node & $4(8 \%)$ \\
Left inguinal lymph node & $5(10 \%)$ \\
Right cervical lymph node & $3(6 \%)$ \\
Intra-parotid lymph node & $1(2 \%)$ \\
Para-aortic lymph node & $3(6 \%)$ \\
Lung & $4(8 \%)$ \\
\hline Subcutis &
\end{tabular}

\subsection{Braf V600E ICC and Molecular Detection}

BRAF ICC was performed on CB sections in all cases. Molecular evaluation of the BRAF status was performed in all cases, particularly on cytological samples in 17 cases and on histological samples in 33 cases (primitive or metastasis). BRAF ICC resulted adequate in $49 / 50(98 \%)$ cases, positive in 15 out of $50(30 \%)$ cases and negative in 34 out of $50(68 \%)$ of cases (Figure 1).

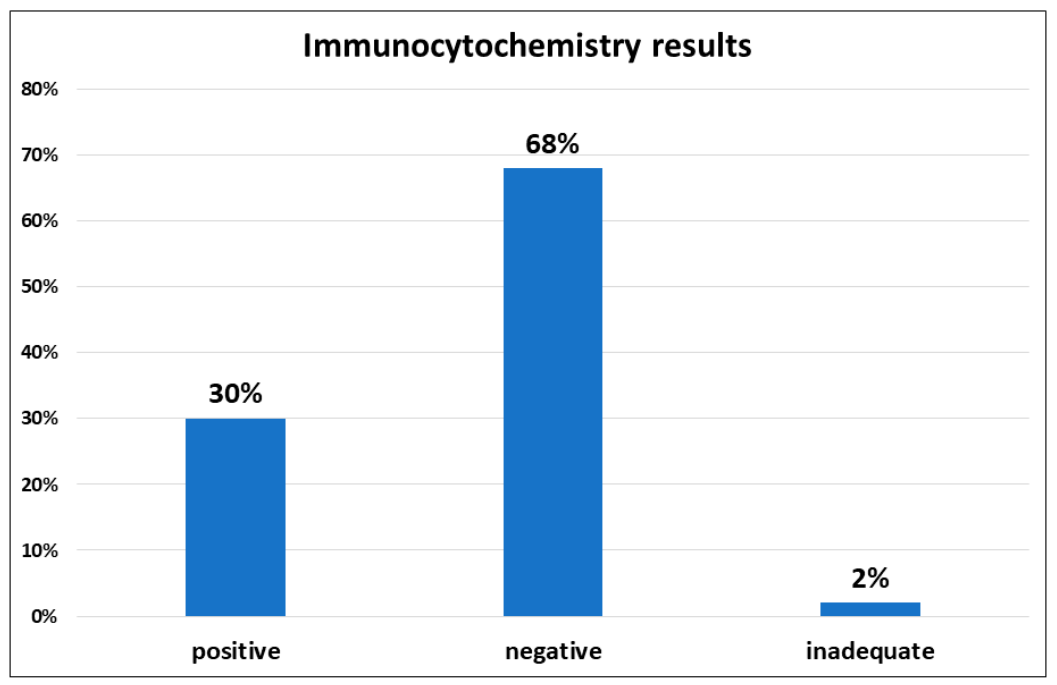

Figure 1. BRAF V600E mutation-specific adequacy. The immunocytochemistry resulted in adequate in $98 \%$, and only 1 out of 50 (2\%) cases resulted in inadequate. BRAF Immunocytochemistry resulted positive in $30 \%$ of the cases and negative in the remaining $68 \%$.

The percentage of positive neoplastic cells ranged from $40 \%$ to $100 \%$, with the mean positivity of $75.3 \%$, and the median positivity of $80 \%$ (Figure 2). The staining intensity resulted heterogeneous, ranging from slight to strong intensity (Figure 3). 


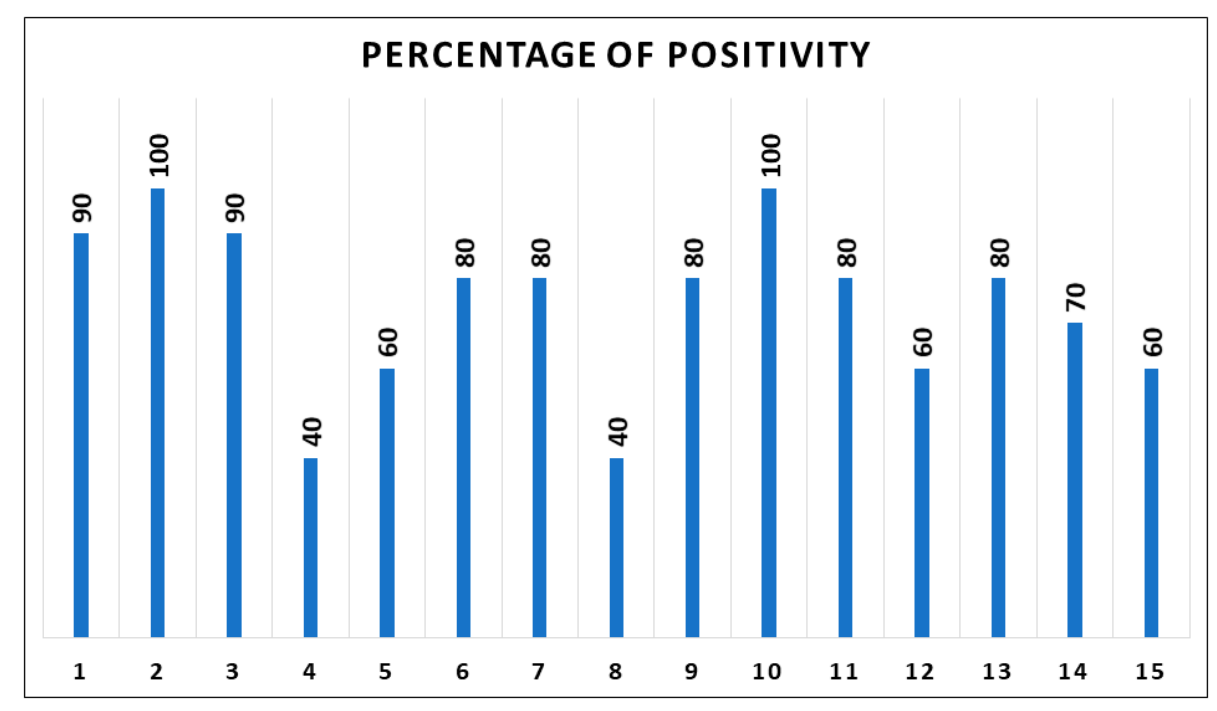

Figure 2. Percentage of BRAF immunocytochemistry-positive cells in the 15 positive cases.
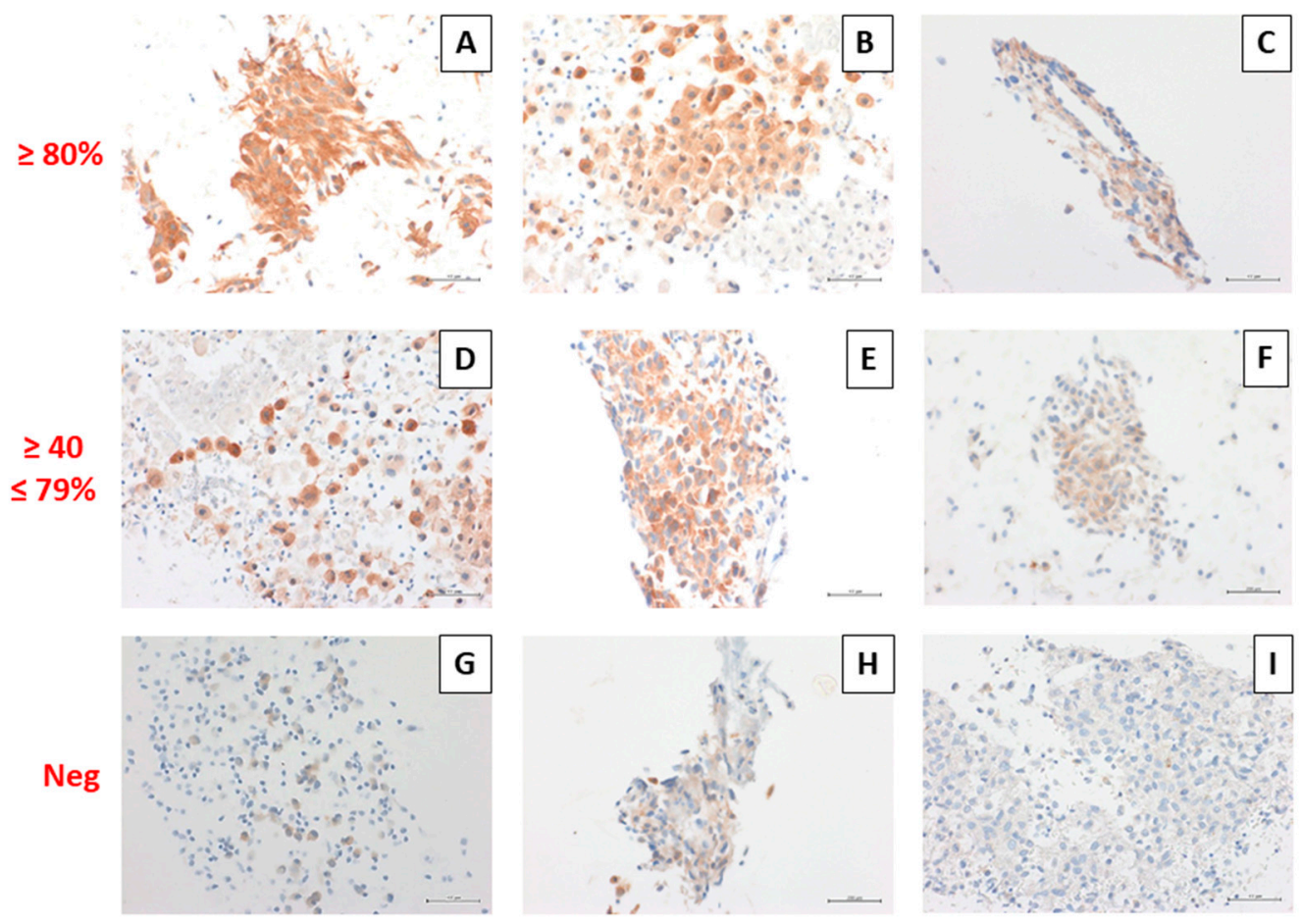

Figure 3. Overview of BRAF V600E immunocytochemistry on CB sections. Granular cytoplasmic positivity with different intensity of the immunocytochemical signal: strong intensity $(\mathbf{A}, \mathbf{D})$, moderate intensity (B,E), slight intensity $(\mathbf{C}, \mathbf{F})$ in over $80 \%$ of the neoplastic cells $(\mathbf{A}-\mathbf{C})$ and between 40 and $79 \%$ of the neoplastic cells (D-F) Negative cases (G-I). (Immunocytochemical stains, original magnification $400 \times$ ).

BRAF V600E mutation was confirmed by molecular analysis in all the 15 BRAF ICCpositive cases. The analysis was performed on the corresponding histological samples ( 2 cases on the primitive neoplasms and 8 cases on the metastatic surgical biopsies) in 10 cases, and on the cytological samples in 5 cases. One out of 15 BRAF ICC-positive cases showed both V600E and V600D mutations at the molecular analysis.

Concerning the 34 negative cases, molecular analysis was performed on corresponding histological samples in 22 cases (14 cases on the primitive neoplasms and 8 cases on the metastatic surgical biopsies) and on cytological samples in 12 cases. The molecular analysis 
confirmed the absence of BRAF V600E mutation in 32 out of 34 (94.1\%) cases. BRAF V600E mutation was instead detected by molecular analysis in 2 out of 34 (5.9\%) ICC-negative cases.

In one case (2\%), the BRAF ICC test was considered inadequate because of the presence of abundant melanin pigment that invalidated the evaluation.

BRAF molecular analysis resulted in inadequate in one case, in which the analysis was performed on a histological sample of the primitive neoplasm. In this case, the cellularity of the cytological sample was not sufficient for the molecular test, and the patient was not submitted to surgery for the removal of the metastases. Moreover, the biological sample of the primitive neoplasm resulted inadequate for the molecular test, as it was a thin CM and the biomaterial was largely consumed for diagnostic tests (multiple levels, immunohistochemical tests).

BRAF mutations different from V600E were detected by molecular analysis in two cases and included V600K in one case and V600R in the other case.

\subsection{Diagnostic Performance of BRAF ICC}

Considering BRAF V600E mutation, overall BRAF ICC showed a SE of $88.2 \%$ and an SP of $100 \%$. The positive predictive value (PPV) was $100 \%$ and the negative predictive value (NPV) was $94.1 \%$. We can define two different sub-groups in the series: (1) BRAF V600E ICC vs. molecular evaluation on histological samples and (2) BRAF V600E ICC vs. molecular evaluation on cytological samples. Indeed, molecular analysis was performed on histological samples when the cytological samples were not sufficient for both diagnostic and predictive purposes.

Diagnostic performance of BRAF ICC resulted differently in these two sub-groups, being higher in the subgroup of cytological samples. Indeed, in the first group, the SE, SP, PPV, and NPV were $83.3 \%, 100 \%, 100 \%$, and $90.9 \%$, respectively. While, in the second group, the SE, SP, PPV and NPV were 100\%, 100\%, 100\% and 100\%, respectively. Considering all the BRAF mutations, and including thus also BRAF mutations other than V600E, SE, SP, PPV and NPV were $78.9 \%, 100 \%, 100 \%$ and $88.2 \%$, respectively.

Results about the diagnostic performance of BRAF ICC are summarized in Table 2.

Table 2. Diagnostic performance of BRAF V600E mutation-specific immunocytochemistry.

\begin{tabular}{cccc}
\hline & $\begin{array}{c}\text { Overall } \\
\text { Series }\end{array}$ & $\begin{array}{c}\text { Molecular Tests on } \\
\text { Histology }\end{array}$ & $\begin{array}{c}\text { Molecular Tests on } \\
\text { Cytology }\end{array}$ \\
\hline Tested cases & 50 & 33 & 17 \\
\hline Adequacy & $49 / 50(98 \%)$ & $32 / 33(96.9 \%)$ & $17 / 17(100 \%)$ \\
\hline True-Positive & 15 & 10 & 5 \\
\hline False-Positive & 0 & 0 & 0 \\
\hline True-Negative & 32 & 20 & 12 \\
\hline False-Negative & 2 & 2 & $100 \%$ \\
\hline Sensitivity & $88.2 \%$ & $83.3 \%$ & $100 \%$ \\
\hline Specificity & $100 \%$ & $100 \%$ & $100 \%$ \\
\hline PPV & $100 \%$ & $100 \%$ & $100 \%$ \\
\hline NPV & $94.1 \%$ & $90.9 \%$ & \\
\hline Accuracy & $95.1 \%$ & $93.7 \%$ & $00 \%$ \\
\hline
\end{tabular}

Abbreviations: PPV: positive predictive value; NPV: negative predictive value.

Some examples of BRAF ICC staining are shown in Figure 4. 

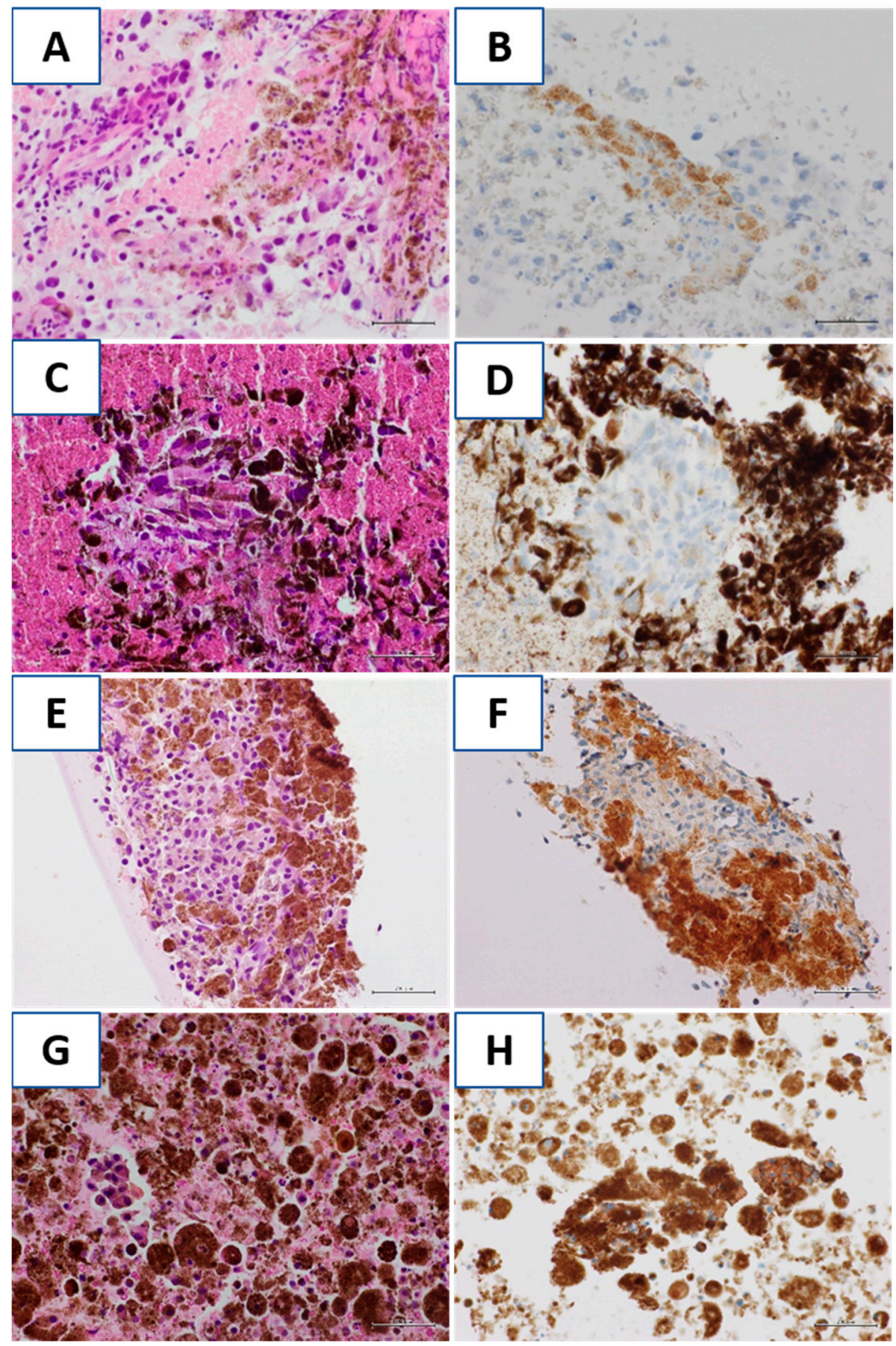

Figure 4. (A,B) Case 1, presence of melanic pigment in the cytoplasm of histiocytes and melanoma cells on cell-block section. BRAF ICC was negative in malignant cells. In this case, at the molecular assessment, BRAF resulted from wild-type; (C,D) Case 2, a melanoma cellular group in a proteinaceous background with melanic pigment on cell-block section. BRAF ICC was negative in the malignant group. At the molecular assessment, BRAF was wild-type; (E,F) Case 3, a cellular aggregate of melanoma cells in a melanic background on cell-block section. BRAF ICC was negative in the neoplastic cells. At the molecular assessment, BRAF V600R mutation was detected; $(\mathbf{G}, \mathbf{H})$ Case 4 , numerous engulfing histiocytes melanic pigment, which is also present in the background and few melanoma cells were present on cell-block section. BRAF ICC was considered not evaluable. At the molecular assessment, a BRAFV600E was detected. (Hematoxylin-eosin and immunocytochemical stains, original magnification $400 \times)$. 


\section{Discussion}

Therapy of advanced CM has dramatically changed in the last few years. Nowadays, MAPK inhibitors and immunotherapy are applied worldwide, significantly improving the overall survival of the patients [24]. Consequently, the evaluation of BRAF mutational status has become a milestone in the management of patients with advanced $\mathrm{CM}$, directly affecting the therapy choice. Indeed, BRAF inhibitors in combination with MEK inhibitors represent the standard treatment for patients with advanced CM carrying BRAF mutations [25]. Overall, BRAF mutations occur in about $50 \%$ of all CMs but are more frequent in CM developing in intermittently sun-exposed skin [24]. The most common BRAF mutation in CM, accounting for about $70-80 \%$ of the mutated cases, is BRAF V600E, a single nucleotide mutation resulting in the substitution of valine with glutamic acid. The V600E-mutated BRAF constitutively activates MEK regardless of RAS signaling. Other less common BRAF mutations may occur in CM, including V600K (accounting for about 5-6\% of cases), with valine replaced by lysine, V600R and V600D [26].

As the mutational status of the neoplasms may be discordant between the primary neoplasms and the metastases in the same patient [18], the actual guidelines recommend defining the mutational status of the metastases, when it is possible [20]. However, the management of the biomaterial is a hot issue in advanced melanoma, and it is often the main limitation for the predictive tests. Indeed, the direct sampling of the metastases is usually unnecessary for the diagnosis, which is usually based on clinical and instrumental findings. On the other hand, the execution of invasive surgery only to obtain biomaterial for predictive purposes may be problematic and not convenient for patients with lowperformance status and deep metastases. In this setting, FNA represents a useful tool in the management of patients with advanced $\mathrm{CM}$, for both diagnostic and predictive purposes. Indeed, FNA may be used to confirm the diagnosis of $\mathrm{CM}$ metastases in patients with a known history of $\mathrm{CM}$ or to obtain the diagnosis in patients with metastases of unknown primary neoplasm, with high sensitivity and specificity [4]. In addition, FNA may be used to collect biomaterial for the predictive evaluation of BRAF mutational status in patients with a clinically known CM metastasis, as recommended also by recent National Comprehensive Cancer Network guidelines [9]. Interestingly, our series included three cases located in the lungs, three cases located in the intra-parotid lymph nodes and one case located in a deep para-aortic lymph node. In all these cases, sampling biomaterial by FNA rather than by surgery certainly resulted in the best options for the patients. However, the amount of available biomaterial in cytological samples may be a limitation for predictive purposes. Indeed, the diagnosis of CM is challenging, and a variable but significant percentage of the biomaterial could be used for the diagnosis. In these circumstances, the cytological sample may not be sufficient for DNA-based molecular techniques for predictive purposes, which are consequently performed on the primary neoplasm, even if the molecular status could be different between primary neoplasm and metastases. In this clinical scenario, ICC could play an important role to obtain information about BRAF V600E mutation in CM metastases using cytological samples not adequate for DNAbased molecular techniques. Although DNA-based molecular techniques are the gold standard to define BRAF mutational status, BRAF ICC was recently introduced as a rapid test to evaluate the presence of BRAF V600E mutation, demonstrating high diagnostic performance in histological samples [11-14]. However, although it is well-known that FNA samples may be used for molecular analysis of BRAF mutations by DNA-based methods, data about the performance of BRAF ICC on CM cytological samples are missing.

We tested BRAF ICC on a series of 50 FNA samples of CM metastases, comparing the ICC results with the gold standard molecular analysis. In this series, we observed 15 out of $50(30 \%)$ ICC-positive cases. All the ICC-positive cases were confirmed by the molecular analysis, and the series did not include false-positive cases, resulting in an SP and a PPV of $100 \%$. Of the 34 ICC-negative cases, 2 cases showed the BRAF V600E mutation by molecular analysis, representing 2 false-negative cases. False negativity could be due to defects of the technique, or to defects of sampling. Overall, BRAF ICC demonstrated a high 
diagnostic performance, with an SE of $88.2 \%$, a SP of $100 \%$, a PPV of $100 \%$ and a NPV of $94.1 \%$. Our results are comparable to the data reported in the literature regarding BRAF ICC applied to histological samples. Indeed, in the studies about diagnostic performance of BRAF ICC performed on histological samples, the SE ranges from $85 \%$ to $100 \%$ and the SP ranges from $93 \%$ to $100 \%[11,12,14,23,27-35]$.

In our series, BRAF ICC interpretation resulted relatively easily in most cases, as the intensity of the staining was high or moderate in 13 out of $15(86.7 \%)$ positive cases. Moreover, a high number of neoplastic cells (often, most of the neoplastic cells) resulted stained in the positive cases, as the least percentage of positive cells was $40 \%$, the mean percentage of positive cells was $75.3 \%$ and the median percentage of positive cells was $80 \%$. However, attention must be paid to the evaluation of the overly-pigmented cases. Indeed, BRAF ICC shows cytoplasmic staining and consequently, the staining may overlap with melanin pigment. Our series included four $(8 \%)$ cases characterized by intense and diffuse pigmentation. For these cases, a collegial evaluation of the BRAF immunostained section and the corresponding $\mathrm{H}$ and $\mathrm{E}$-stained section of the corresponding $\mathrm{CB}$ was performed. In three of the four cases, the evaluation of the immunocytochemical signal was possible thanks to the presence on the immunostained sections of clearly negative malignant cell groups (Figure 4).

For these cases, the molecular evaluation confirmed the negativity of the test. In the fourth case, the abundant melanic pigment in the cytoplasm and in the background, together with a discohesive pattern, did not allow a clear evaluation of the immunocytochemical signal and therefore this case was considered inadequate for ICC evaluation (Figure 5). Molecular analysis should always be considered in heavily pigmented cases, to avoid the risk of false-positive results.

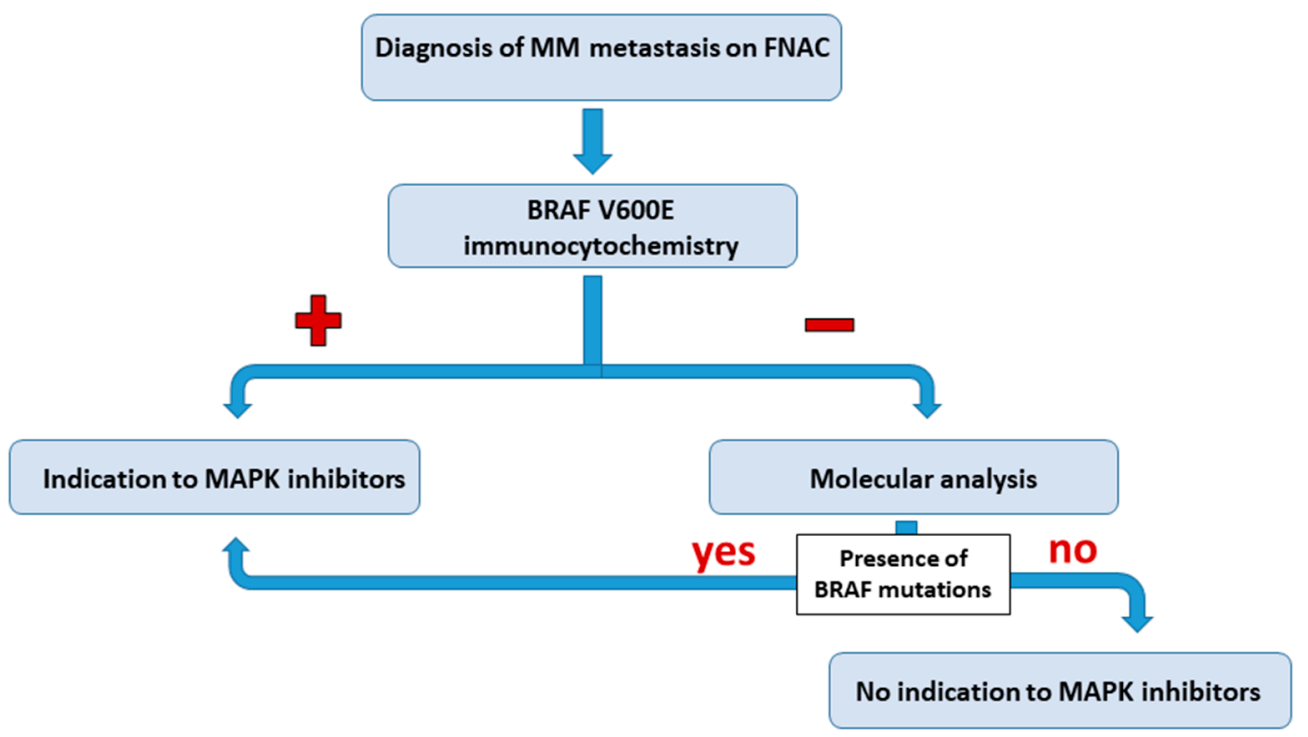

Figure 5. Proposed algorithm for the evaluation of the BRAF V600E mutation on a cytological sample.

Moreover, it should be kept in mind that BRAF ICC only stains cases harboring V600E mutation, but it does not stain cases harboring other BRAF mutations. In our series, indeed, BRAF mutation other than V600E results were present in two cases (one case with V600K mutation and one case with both V600K and V600R mutations). Considering the possibility of BRAF ICC false-negative cases, and the possibility of BRAF mutations other than V600E in ICC negative cases, BRAF ICC should be applied to FNA samples as a screening tool, and molecular analysis should be considered mandatory in BRAF ICC negative cases. We propose an algorithm in which BRAF ICC represents the first step for the evaluation of BRAF mutational status in cytological samples. A positive result could be adequate to 
address the patient to target therapy, while molecular analysis should be performed in BRAF ICC negative cases (Figure 5).

This algorithm may be particularly useful in poorly cellular samples. Indeed, the cytological samples sometimes may be poorly cellular, and consequently not suitable for extraction-based molecular analysis, forcing the clinician to obtain a surgical excision of the metastasis, or the pathologists to perform the predictive tests on the primitive neoplasms. Moreover, even in the primitive melanoma, the neoplastic component could be scant, making the identification of BRAF mutation unsuccessful. In these settings, the chance offered by ICC on cytological samples from CM metastases could be a valid opportunity for the therapeutic strategy of the patients.

\section{Conclusions}

In conclusion, BRAF ICC is a rapid, cost-effective method for detecting BRAF V600E mutation in MM metastases, applicable with high diagnostic performance to cytological samples. It could represent the first step to evaluate BRAF mutational status in cytological samples, mainly in poorly cellular cases.

Author Contributions: Conceptualization, A.R. and I.C.; Data curation, G.A., G.B., S.N. and R.F.; Formal analysis, E.M.; Investigation, A.R., M.C., A.G., T.T. and I.C.; Cytological slides review and evaluation, I.C., M.M.; Methodology, M.M., F.Z.M. and I.C.; Supervision, R.F.; Writing—original draft, A.R. and I.C.; Writing-review \& editing, I.C. All authors have read and agreed to the published version of the manuscript.

Funding: This research received no external funding.

Institutional Review Board Statement: The study was conducted according to the guidelines of the Declaration of Helsinki and approved by the Institutional Ethics Committee of University "Vanvitelli" (protocol code 13375; date of approval 3 June 2020).

Informed Consent Statement: Informed consent was obtained from all subjects involved in the study.

Data Availability Statement: The data presented in this study are available on request from the corresponding author.

Conflicts of Interest: The authors declare no conflict of interest.

\section{References}

1. Bray, F.; Ferlay, J.; Soerjomataram, I.; Siegel, R.L.; Torre, L.A.; Jemal, A. Global cancer statistics 2018: GLOBOCAN estimates of incidence and mortality worldwide for 36 cancers in 185 countries. CA Cancer J. Clin. 2018, 68, 394-424. [CrossRef]

2. Tripp, M.K.; Watson, M.; Balk, S.J.; Swetter, S.M.; Gershenwald, J.E. State of the science on prevention and screening to reduce melanoma incidence and mortality: The time is now. CA Cancer J. Clin. 2016, 66, 460-480. [CrossRef]

3. Hall, B.J.; Schmidt, R.L.; Sharma, R.R.; Layfield, L.J. Fine-needle aspiration cytology for the diagnosis of metastatic melanoma. Am. J. Clin. Pathol. 2013, 140, 635-642. [CrossRef]

4. Ronchi, A.; Zito Marino, F.; Toni, G.; Pagliuca, F.; Russo, D.; Signoriello, G.; Moscarella, E.; Brancaccio, G.; Argenziano, G.; Franco, R.; et al. Diagnostic performance of melanocytic markers for immunocytochemical evaluation of lymph-node melanoma metastases on cytological samples. J. Clin. Pathol. 2021. [CrossRef]

5. Herbert, A.; Koo, M.M.; Barclay, M.E.; Greenberg, D.C.; Abel, G.A.; Levell, N.J.; Lyratzopoulos, G. Stage-specific incidence trends of melanoma in an English region, 1996-2015: Longitudinal analyses of population-based data. Melanoma Res. 2020, 30, $279-285$. [CrossRef]

6. Kunz, M.; Vera, J. Modelling of protein kinase signaling pathways in melanoma and other cancers. Cancers 2019, 11, 465. [CrossRef] [PubMed]

7. Greaves, W.O.; Verma, S.; Patel, K.P.; Davies, M.A.; Barkoh, B.A.; Galbincea, J.M.; Yao, H.; Lazar, A.J.; Aldape, K.D.; Medeiros, L.J.; et al. Frequency and spectrum of BRAF mutations in a retrospective, single-institution study of 1112 cases of melanoma. JMD 2013, 15, 220-226. [CrossRef]

8. Rubinstein, J.C.; Sznol, M.; Pavlick, A.C.; Ariyan, S.; Cheng, E.; Bacchiocchi, A.; Kluger, H.M.; Narayan, D.; Halaban, R. Incidence of the V600K mutation among melanoma patients with BRAF mutations, and potential therapeutic response to the specific BRAF inhibitor PLX4032. J. Transl. Med. 2010, 8, 67. [CrossRef] [PubMed]

9. NCCN-National Comprehensive Cancer. Available online: http://www.nccn.org/index.asp (accessed on 17 June 2021). 
10. Lombardi, A.; Russo, M.; Luce, A.; Morgillo, F.; Tirino, V.; Misso, G.; Martinelli, E.; Troiani, T.; Desiderio, V.; Papaccio, G.; et al. Comparative study of NGS platform Ion Torrent personal genome machine and Therascreen Rotor-Gene $\mathrm{Q}$ for the detection of somatic variants in cancer. High Throughput 2020, 9, 4. [CrossRef] [PubMed]

11. Capper, D.; Preusser, M.; Habel, A.; Sahm, F.; Ackermann, U.; Schindler, G.; Pusch, S.; Mechtersheimer, G.; Zentgraf, H.; von Deimling, A. Assessment of BRAF V600E mutation status by immunohistochemistry with a mutation-specific monoclonal antibody. Acta Neuropathol. 2011, 122, 11-19. [CrossRef]

12. Long, G.V.; Wilmott, J.S.; Capper, D.; Preusser, M.; Zhang, Y.E.; Thompson, J.F.; Kefford, R.F.; von Deimling, A.; Scolyer, R.A. Immunohistochemistry is highly sensitive and specific for the detection of V600E BRAF mutation in melanoma. Am. J. Surg. Pathol. 2013, 37, 61-65. [CrossRef]

13. Schirosi, L.; Strippoli, S.; Gaudio, F.; Graziano, G.; Popescu, O.; Guida, M.; Simone, G.; Mangia, A. Is immunohistochemistry of BRAF V600E useful as a screening tool and during progression disease of melanoma patients? BMC Cancer 2016, 16, 905. [CrossRef] [PubMed]

14. Feller, J.K.; Yang, S.; Mahalingam, M. Immunohistochemistry with a mutation-specific monoclonal antibody as a screening tool for the BRAFV600E mutational status in primary cutaneous malignant melanoma. Mod. Pathol. 2013, 26, 414-420. [CrossRef] [PubMed]

15. Vitagliano, G.; Santoro, G.; Landolfi, L.; Cozzolino, I.; Peluso, A.L.; Ieni, A.; Selleri, C.; Zeppa, P. Fine-needle cytology of intraglandular parotid lymph node: A useful procedure in the management of salivary gland nodules. Diagn. Cytopathol. 2019, 47, 695-700. [CrossRef] [PubMed]

16. Chen, S.; Randolph, M.; Cramer, H.M.; Watkins, T.; McCullough, H.; Post, K.M.; Sen, J.D.; Cheng, L.; Wu, H.H. Detection of BRAF mutation in metastatic melanoma utilizing cell-transferred cytological smears. Acta Cytol. 2014, 58, 478-482. [CrossRef] [PubMed]

17. Bernacki, K.D.; Betz, B.L.; Weigelin, H.C.; Lao, C.D.; Redman, B.G.; Knoepp, S.M.; Roh, M.H. Molecular diagnostics of melanoma fine-needle aspirates: A cytology-histology correlation study. Am. J. Clin. Pathol. 2012, 138, 670-677. [CrossRef]

18. Valachis, A.; Ullenhag, G.J. Discrepancy in BRAF status among patients with metastatic malignant melanoma: A meta-analysis. Eur. J. Cancer 2017, 81, 106-115. [CrossRef]

19. Pellegrini, C.; Di Nardo, L.; Cipolloni, G.; Martorelli, C.; De Padova, M.; Antonini, A.; Maturo, M.G.; Del Regno, L.; Strafella, S.; Micantonio, T.; et al. Heterogeneity of BRAF, NRAS, and TERT promoter mutational status in multiple melanomas and association with MC1R genotype: Findings from molecular and immunohistochemical analysis. J. Mol. Diagn. JMD 2018, 20, 110-122. [CrossRef]

20. Michielin, O.; van Akkooi, A.C.J.; Ascierto, P.A.; Dummer, R.; Keilholz, U.; ESMO Guidelines Committee. Electronic address: Clinicalguidelines@esmo.org. Cutaneous melanoma: ESMO Clinical Practice Guidelines for diagnosis, treatment and follow-up. Ann. Oncol. 2019, 30, 1884-1901. [CrossRef]

21. Da Cunha Santos, G.; Saieg, M.A. Preanalytic specimen triage: Smears, cell blocks, cytospin preparations, transport media, and cytobanking. Cancer Cytopathol. 2017, 125, 455-464. [CrossRef]

22. Manca, A.; Paliogiannis, P.; Colombino, M.; Casula, M.; Lissia, A.; Botti, G.; Caracò, C.; Ascierto, P.A.; Sini, M.C.; Palomba, G.; et al. Mutational concordance between primary and metastatic melanoma: A next-generation sequencing approach. J. Transl. Med. 2019, 17, 289. [CrossRef] [PubMed]

23. Boursault, L.; Haddad, V.; Vergier, B.; Cappellen, D.; Verdon, S.; Bellocq, J.P.; Jouary, T.; Merlio, J.P. Tumor homogeneity between primary and metastatic sites for BRAF status in metastatic melanoma determined by immunohistochemical and molecular testing. PLoS ONE 2013, 8, e70826. [CrossRef] [PubMed]

24. Shtivelman, E.; Davies, M.Q.; Hwu, P.; Yang, J.; Lotem, M.; Oren, M.; Flaherty, K.T.; Fisher, D.E. Pathways and therapeutic targets in melanoma. Oncotarget 2014, 5, 1701-1752. [CrossRef] [PubMed]

25. Queirolo, P.; Spagnolo, F. BRAF plus MEK-targeted drugs: A new standard of treatment for BRAF-mutant advanced melanoma. Cancer Metastasis Rev. 2017, 36, 35-42. [CrossRef] [PubMed]

26. Davies, H.; Bignell, G.R.; Cox, C.; Stephens, P.; Edkins, S.; Clegg, S.; Teague, J.; Woffendin, H.; Garnett, M.J.; Bottomley, W.; et al. Mutations of the BRAF gene in human cancer. Nature 2002, 417, 949-954. [CrossRef] [PubMed]

27. Pearlstein, M.V.; Zedek, D.C.; Ollila, D.W.; Treece, A.; Gulley, M.L.; Groben, P.A.; Thomas, N.E. Validation of the VE1 immunostain for the BRAF V600E mutation in melanoma. J. Cutan. Pathol. 2014, 41, 724-732. [CrossRef] [PubMed]

28. Skorokhod, A.; Capper, D.; von Deimling, A.; Enk, A.; Helmbold, P. Detection of BRAF V600E mutations in skin metastases of malignant melanoma by monoclonal antibody VE1. J. Am. Acad. Dermatol. 2012, 67, 488-491. [CrossRef]

29. Routhier, C.A.; Mochel, M.C.; Lynch, K.; Dias-Santagata, D.; Louis, D.N.; Hoang, M.P. Comparison of 2 monoclonal antibodies for immunohistochemical detection of BRAF V600E mutation in malignant melanoma, pulmonary carcinoma, gastrointestinal carcinoma, thyroid carcinoma, and gliomas. Hum. Pathol. 2013, 44, 2563-2570. [CrossRef]

30. Lade-Keller, J.; Kristensen, L.S.; Riber-Hansen, R.; Guldberg, P.; Hansen, L.L.; Steiniche, T.; Hager, H. A role for immunohistochemical detection of BRAF V600E prior to BRAF-inhibitor treatment of malignant melanoma? J. Clin. Pathol. 2013, 66, 723-725. [CrossRef]

31. Hofman, V.; Ilie, M.; Long-Mira, E.; Giacchero, D.; Butori, C.; Dadone, B.; Selva, E.; Tanga, V.; Passeron, T.; Poissonnet, G.; et al. Usefulness of immunocytochemistry for the detection of the BRAF(V600E) mutation in circulating tumor cells from metastatic melanoma patients. J. Investig. Dermatol. 2013, 133, 1378-1381. [CrossRef] [PubMed] 
32. Ihle, M.A.; Fassunke, J.; König, K.; Grünewald, I.; Schlaak, M.; Kreuzberg, N.; Tietze, L.; Schildhaus, H.U.; Büttner, R.; MerkelbachBruse, S. Comparison of high resolution melting analysis, pyrosequencing, next generation sequencing and immunohistochemistry to conventional Sanger sequencing for the detection of p.V600E and non-p.V600E BRAF mutations. BMC Cancer 2014, 14, 13. [CrossRef] [PubMed]

33. Colomba, E.; Hélias-Rodzewicz, Z.; Von Deimling, A.; Marin, C.; Terrones, N.; Pechaud, D.; Surel, S.; Côté, J.F.; Peschaud, F.; Capper, D.; et al. Detection of BRAF p.V600E mutations in melanomas: Comparison of four methods argues for sequential use of immunohistochemistry and pyrosequencing. J. Mol. Diagn. 2013, 15, 94-100. [CrossRef] [PubMed]

34. Busam, K.J.; Hedvat, C.; Pulitzer, M.; von Deimling, A.; Jungbluth, A.A. Immunohistochemical analysis of BRAF(V600E) expression of primary and metastatic melanoma and comparison with mutation status and melanocyte differentiation antigens of metastatic lesions. Am. J. Surg. Pathol. 2013, 37, 413-420. [CrossRef]

35. Chen, Q.; Xia, C.; Deng, Y.; Wang, M.; Luo, P.; Wu, C.; Yue, J.; Fang, N.; Wang, M.; Wei, S. Immunohistochemistry as a quick screening method for clinical detection of BRAF(V600E) mutation in melanoma patients. Tumor Biol. 2014, 35, 5727-5733. [CrossRef] [PubMed] 